\title{
Towards biologically constrained attractor models of schizophrenia
}

Short title: Constraining attractor models of schizophrenia

Heike Stein ${ }^{1^{*}}$, Joao Barbosa ${ }^{1^{*}}$, Albert Compte ${ }^{2}$

${ }^{1}$ Laboratoire de Neurosciences Cognitives et Computationnelles, Département d'Études Cognitives, École Normale Supérieure, INSERM U960, PSL University, Paris, France

${ }^{2}$ Institut d'Investigacions Biomèdiques August Pi i Sunyer (IDIBAPS), Barcelona, Spain

* These authors contributed equally to this work.

Correspondence: Albert Compte, IDIBAPS, C. Rosselló 149-153, 08036 Barcelona, Spain, acompte@clinic.cat

Declarations of interest: none

\section{Highlights}

- People with schizophrenia have quantitative working memory (WM) deficits

- WM deficits cannot be readily mapped on the stability of continuous attractors

- Different attractor models yield inconsistent behavioral predictions upon changes in E/I ratio

- Grouping different perturbations under E/I ratio can be inadequate for some deficits

- Insufficient experimental and computational understanding precludes model selection

- We need data showing NMDAR-dependence of rate tuning and correlated noise in WM circuits

\begin{abstract}
Alterations in neuromodulation or synaptic transmission in biophysical attractor network models, as proposed by the dominant dopaminergic and glutamatergic theories of schizophrenia, successfully mimic working memory (WM) deficits in people with schizophrenia (PSZ). Yet, multiple, often opposing circuit mechanisms can lead to the same behavioral patterns in these network models. Here, we critically revise the computational and experimental literature that links NMDAR hypofunction to WM precision loss in PSZ. We show in network simulations that currently available experimental evidence cannot set apart competing mechanistic accounts, and critical points to resolve are firing rate tuning and shared noise modulations by E/l ratio alterations through NMDAR blockade, and possible concomitant deficits in short-term plasticity. We argue that these concerted experimental and computational efforts will lead to a better understanding of the neurobiology underlying cognitive deficits in PSZ.
\end{abstract}

\section{Keywords}

Schizophrenia, working memory, attractor network, NMDAR, ketamine, short-term synaptic plasticity 
Computational models have been increasingly used to link molecular and cellular mechanisms with systemic and behavioral alterations in psychiatric diseases, as part of the new field of computational psychiatry [1-3]. Dynamical network accounts of brain disease reach from models of distributed processing across brain areas to biophysical models of the local cortical circuitry $[4,5]$. Computational models contribute quantitative perspectives and mechanistic hypotheses about pathological brain functions that have a transformative potential on psychiatric research [6]. However, advancing towards valid model-based links between mechanisms and symptoms will require a concerted effort of theoretical and neurophysiological research to select among currently underconstrained computational models. We illustrate this point here by focusing on circuit models of association cortices that reproduce working memory (WM) alterations in people with schizophrenia (PSZ) (Fig. 1a and Box 1; for a comprehensive review on computational models of schizophrenia, see [7] and [1-3]). These specific models have attracted attention for combining rich quantitative descriptions of multiple behavioral deficits in PSZ with detailed, biologically plausible mechanisms (Box 1), informed by neurophysiological data from behaving animals [8-11] and in vitro experiments $[12,13]$.

(a)

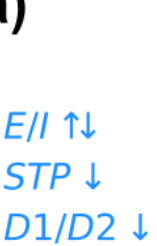

precision
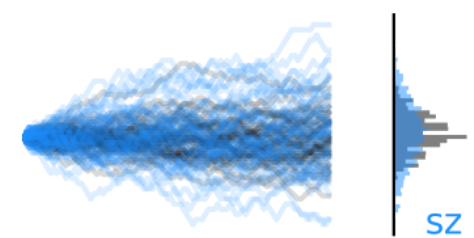

ctrl

\section{capacity}

$E / / \downarrow$

$D 1 / D 2 \downarrow$
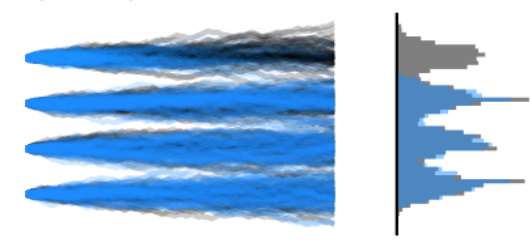

(b)

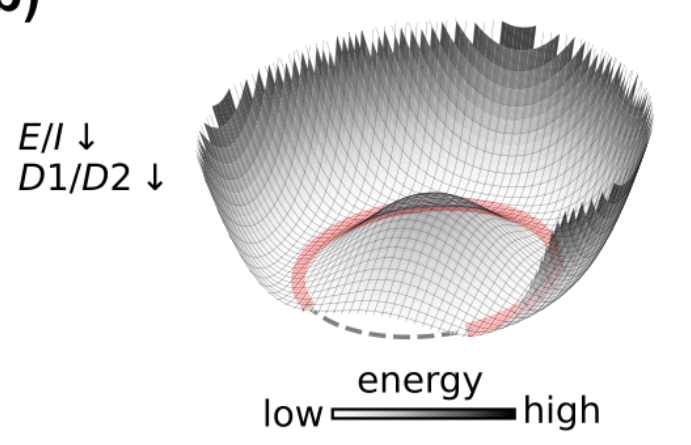

distractibility

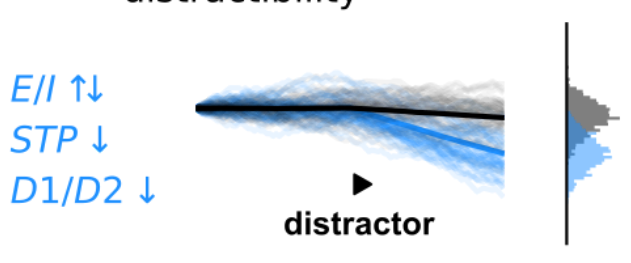

serial dependence
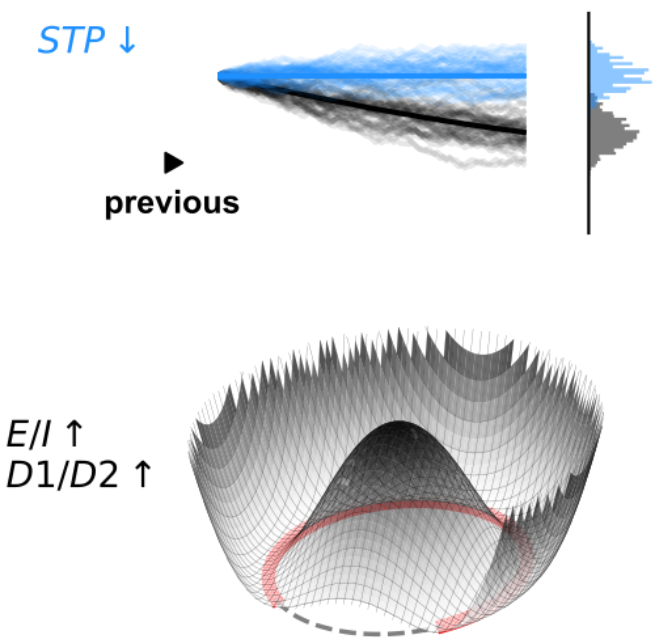

Figure 1. (a) Four different quantifiable alterations in spatial WM in PSZ (black: control simulations; blue: schizophrenia (SZ) simulations), with indication of biophysical network mechanisms that have been proposed to underlie them in computational models (Box 1). $\uparrow$ : increase; $\downarrow$ : decrease; E/l: excitation-inhibition ratio, typically modelled through NMDAR hypofunction at pyramidal cells $(E / / \downarrow)$ or at inhibitory interneurons $(E / I \uparrow)$, but other receptor manipulations could similarly affect network dynamics (e.g. GABA ${ }_{A} R$ dysfunction); STP: short-term plasticity; D1/D2: relative D1- vs. D2-receptor contributions in the dopaminergic system. (b) In continuous attractor models, the deepening or shallowing of WM attractors does not determine the effects of noise, which act on an orthogonal 
space (red manifold). Different angular positions on this low-dimensional manifold encode different stimulus values.

\section{Attractor stability as a general framework in computational psychiatry}

On the basis of these computational models, it has been argued that psychiatric symptoms, and especially those occurring in PSZ, may be more naturally mapped to distortions of network dynamics, rather than directly to specific cellular or molecular mechanisms $[9,14,15]$. For the case of WM, this corresponds to the stability of mnemonic attractor states, i.e. patterns of sustained network activity that represent memoranda. In this framework, different altered neurobiological mechanisms may convergently affect the stability of attractor states (Fig. 1b) and determine changes in network output consistent with disease symptoms (e.g. weaker memories or higher distractibility, Box 1). Results from multiple modeling studies converge on a general framework for WM-related network dynamics in PSZ. In brief, the erosion of the attractor landscape would lead to less stable memories, lower WM capacity, and increased distractibility [14,16-18]. Detailed biophysical models have shown how this same dynamic situation, consistent conceptually with the symptoms of PSZ, can be attained through different mechanistic alterations: from synaptic receptor modulations that alter the excitation-to-inhibition ratio (E/I ratio) of the network [19], complex neuromodulatory actions of the dopamine system on synaptic and cellular channels [20], or the effect of serotonergic receptors on the excitability of network neurons [21] (Box 1 for more references). In turn, a given alteration of network dynamics may be mitigated by "treatment" manipulations unrelated to the initial mechanistic deficit.

The specific mapping of a given mechanistic alteration (e.g. depleting NMDA receptors (NMDARs)) and the resulting network dynamics effect (e.g. weaker attractor states) may in some cases be difficult to establish. In great part, this is due to our currently limited knowledge about the neurophysiology of the implicated cortical circuits and the still insufficient understanding of biological network models. We illustrate this point in the following by focusing on the lower precision of WM reports in PSZ (Box 1) and how this may be explained by a reduction in glutamatergic NMDAR efficacy or in short-term plasticity (STP), which in turn lead to reduced stability of attractors in the face of random fluctuations. Current computational models establish an ambiguous correspondence between this behavioral phenomenon and NMDAR-mediated changes in E/l ratio, which needs further neurophysiological data to be clarified. In contrast, the link between STP at recurrent excitatory synapses and WM precision appears robust in currently plausible network implementations.

\section{E/I ratio has ambiguous effects on WM stability, depending on network biophysics}

From a biological perspective, most evidence points to an increased cortical E/I ratio in PSZ [22-24], possibly caused by hypofunctional NMDARs in inhibitory interneurons (i.e. cortical disinhibition) [12,25,26], (but see [27]). Indeed, computational models that implement cortical disinhibition in WM circuits can explain reduced WM precision and increased distractibility $[19,28,29]$ (but note similar modeling work that instead explains altered decision making biases under ketamine with cortical disexcitation, rather than cortical disinhibition [30]). This occurs through stronger bumps of activity (bump attractors), i.e. higher network activation and deeper attractors. The finding that deeper attractors are more sensitive to noisy fluctuations (resulting in reduced WM precision) is in apparent contradiction with the general framework defined above, where deeper attractors are associated with more stable memories. The apparent paradox is resolved by noting that random displacements of a 
continuous bump attractor occur along the dimension of marginal stability (pink manifold in Fig. 1B), so they are not specifically linked to the depth of the attractor but instead depend rather intricately on the shape of the bump attractor and the amplitude and correlations of noise in the network (see below). The fact that we do not know how NMDAR blockade affects these elements both in the brain and in biologically realistic computational models is at the heart of our current inability to unambiguously evaluate the plausibility of the glutamatergic explanation for a drop in WM precision in PSZ, as we show in the following.

Mathematical analyses of neural field models show that stronger bumps (wider and/or taller) diffuse more slowly, but this can be offset by an increase in noise amplitude or spatial correlation in the network [31,32]. The diffusivity $(D)$ of the bump attractor is proportional to the noise strength $(\varepsilon)$ and the spatial modulation of noise correlations $(\Delta C)$, and depends inversely on the squared amplitude $\left(A^{2}\right)$ of the bump [32,33]:

$$
D \sim \frac{\varepsilon \cdot \Delta C}{A^{2}}
$$

As a result, the specific effect of reduced NMDAR efficacy, or any other manipulation of a given biophysical parameter, on WM diffusion will depend on how the manipulation affects each of these three factors quantitatively. These effects, in turn, are determined by biophysical details of the models, including the origin of correlated noisy fluctuations during the delay period. Only recently have some studies started to address the generation of correlated variability in spatially extended balanced networks [34-36], but more work is needed to fully understand how it changes with specific manipulations of biological parameters.

Specific bump attractor network implementations illustrate the indecisiveness of current modeling accounts of WM precision in PSZ. We simulated two distinct, biophysical bump attractor models which differ fundamentally in the way that noise is generated in the network. In Model 1, noise is injected from external sources, and all-to-all coupling between neurons generates strong noise correlations [29]. This model is similar to the model used in [19], but additionally incorporates STP. Model 2 also includes STP, but its noise is generated internally, by virtue of sparse network connectivity that induces very weak correlations between the noise experienced by different neurons [37]. In Model 1, manipulations of NMDAR efficacy did not affect bump shape strongly (Fig. 2a, top) but in turn had an important effect both on current noise amplitude (Fig. 2b, top) and on the spatial modulation of noise correlations (Fig. 2c, top). In contrast, manipulations of NMDARs in Model 2 had a strong impact on bump shape (Fig. 2a, bottom) and a more modest influence on the noise amplitude (Fig. 2b, bottom) and on the spatial modulation of noise correlations (Fig. 2c, bottom). Repeated simulations show that corresponding perturbations in NMDARs (either on excitatory cells or on inhibitory cells) in these two biophysical models led to opposing predictions for bump diffusion. Disinhibition $(\mathrm{E} \rightarrow \downarrow \downarrow)$ led to faster diffusion in Model 1 (Fig. 2d, top, similar to previous models in $[19,28]$ ), but resulted in slower diffusion in Model 2 (Fig. $2 \mathrm{~d}$, bottom). Importantly, the combination of the three elements defined before (Fig. 2a-c), as dictated by the mathematical formula of the diffusivity (Eq. 1, [32]), explained these differences quantitatively (Fig. 2d, dotted lines). Thus, the direction in which alterations of the E/I ratio through NMDAR hypofunction affect WM precision will depend on the biophysical 
details of the WM-maintaining circuit, and on how these mechanisms affect bump shape, noise amplitude and the spatial modulation of noise correlations. Which biophysical mechanisms affect bump shape and noise characteristics is currently unclear, but candidate mechanisms that distinguish the two models include: sparseness of connectivity, strength of external noisy inputs, saturation of NMDARs, conductance-based synapses, and oscillatory activity.

(a)
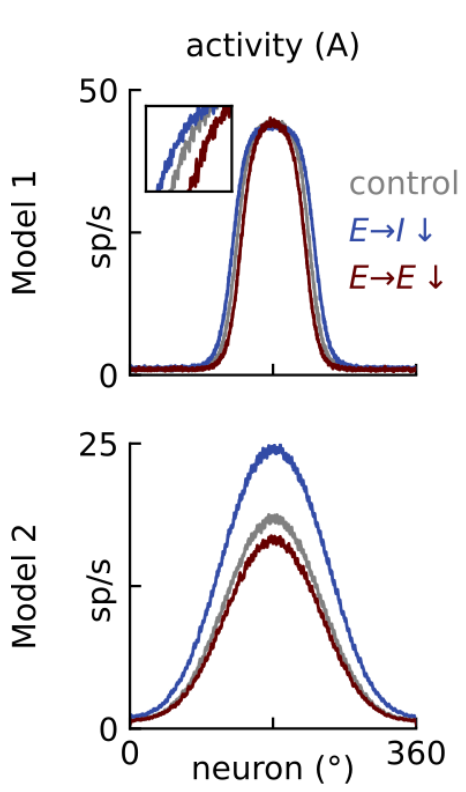

(b)
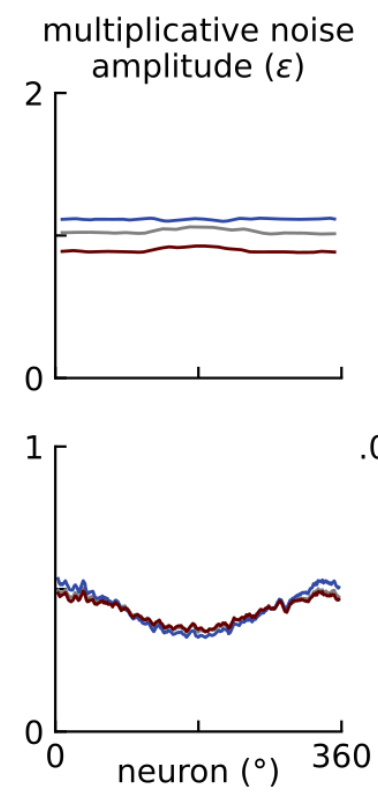

(c)
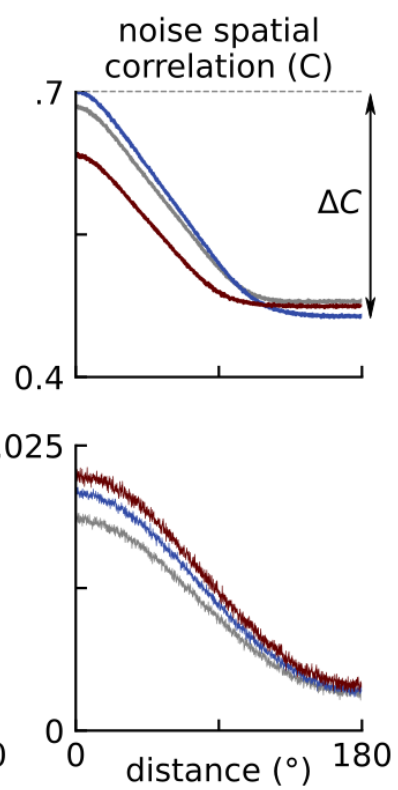

(d)
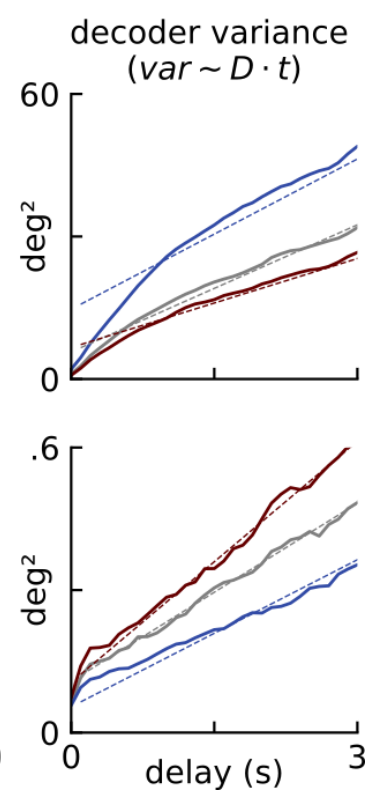

Figure 2. Different biophysical network models yield opposing predictions on how E/l ratio affects WM precision. We tested two different biophysical models of spatial WM. Model 1 , upper panels: all-to-all connectivity, conductance-based synapses, saturating NMDARs [29]; Model 2, lower panels: sparse connectivity, current-based synapses, non-saturating NMDARs [37]. E/l ratio was modulated by reducing the efficacy of NMDARs on excitatory neurons $(E \rightarrow E \downarrow$, resulting in reduced $E / l$ ratio, $E / I \downarrow)$ or on inhibitory neurons $(E \rightarrow \mid \downarrow$, causing $E / / \uparrow)$, mimicking possible effects of NMDAR antagonists. The two network models responded very differently to these manipulations: with little effect on bump strength and strong impact on noise parameters (model 1) or with strong impact on bump shape but minor effects on noise parameters (model 2). As a result, diffusivity of memory encoding increased with $\mathrm{E} / \mathrm{l}$ ratio for model 1 but decreased with $\mathrm{E} / \mathrm{l}$ ratio for model 2 , in agreement with mathematical derivations of how diffusivity depends on bump strength $(A)$, noise amplitude $(\varepsilon)$ and the modulation of the spatial correlation of noisy fluctuations $(\Delta C)$ (formula inset [32], predicting red and blue slopes in dotted straight lines, intercepts and gray slopes were independently fitted to simulation data). (a) population activity during delay for simulation with cue stimulus at $180^{\circ}$; (b) coefficient of variation (std/mean) of total synaptic current into excitatory neurons ( $1 \mathrm{~s}$ during delay) shows approximately flat tuning in the models, consistent with the multiplicative noise condition in [32]; (c) spatial correlation of fluctuations around the mean for the total synaptic current in the delay (1 $s$ window). Note the large quantitative difference between the two models, due to dense versus sparse connectivity [36]. Of significance for bump diffusivity is the modulation of the spatial correlation $\Delta C$; (d) memory diffusion computed as the time-by-time variance of population vector decoding of bump centers (250-ms windows) for 1,250 different simulations in 3-s-long delay periods. For model 2 , systematic drifts [37] were removed by subtracting the mean decoder trajectory of 25 simulations with identical cue stimulus, and for 50 different cue stimuli, before computing the variance. For visualization purposes, high heterogeneity in model 2 was smoothed with a square kernel in a-c. Details of network simulations and analyses are available at https://github.com/comptelab/attractorSZ. 


\section{Insufficient and inconsistent experimental constraints on plausible computational models}

The detailed understanding of how a diversity of plausible network models (including the two models highlighted above) react to mechanistic manipulations can be exploited to identify the regime in which cortical circuits operate, and motivate neurophysiological and pharmacological experiments in behaving animals. A recent study that raised the E/I ratio in monkeys through cholinergic neuromodulation by microstimulation of nucleus basalis shows that WM precision increases, and bump attractors in the prefrontal cortex become wider [38]. This represents an experimental validation of the impact of bump attractor shape on WM diffusivity according to Eq. 1. However, a recent study under ketamine showed a similar reduction in delay-period tuning in prefrontal cortex, but this time associated with decreased precision [39], which suggests that manipulation of NMDARs may have an important impact on noise parameters $[40,41]$ that compensate for the influence of bump shape on diffusivity (as for Model 1 in Fig. 2). The opposing effects of E/I ratio on WM performance in [38,39] suggest that the convenient interpretation of functional alterations as the effect of modulations in E/l ratio, via distinct convergent mechanistic manipulations, may be an inadequate simplification of the mechanistic basis of cognitive deficits of PSZ. Future experiments with these and other neuromodulators or receptor antagonists should explicitly assess the impact on bump shape and correlated noise during delay activity, together with WM precision, in order to clarify the validity of E/l ratio as a useful construct for the interpretation of these data.

It is useful here to consider the administration of systemic NMDAR antagonists as a pharmacological model of schizophrenia [42]. Mimicking effects in PSZ, NMDAR antagonists cause reduced accuracy in WM tasks $[10,24,39,43]$, possibly reflecting a drop in WM precision. Despite the dominant view that systemic ketamine generally raises E/l ratio and disinhibits cortical networks [11,25,26,44], more specific evidence indicates that in the context of WM tasks, prefrontal delay activity is often suppressed in PSZ $[45,46]$ and under systemic NMDAR blockade $[10,39,43,47,48]$ (but see [40]). However, some of the most compelling evidence for NMDAR-blockade-related suppression of delay activity in prefrontal cortex comes from iontophoresis studies, which affect receptors only in the very near neighborhood of the electrode tip [10,49]. Using computational network models, we illustrate how the suppressive effects of iontophoresis cannot be unequivocally associated with the network effects of systemic administration (Fig. 3). Indeed, when NMDAR antagonism is comparatively more effective on interneuron receptors than on pyramidal neuron receptors, a paradoxical effect emerges where delay activity is suppressed in iontophoresis simulations (Fig. 3c-d), but enhanced in whole-network application simulations (Fig. 3b). Electrophysiology studies in monkeys performing a WM task under systemic NMDAR antagonists have mostly found decreased [10,39,47] but also increased [40] prefrontal mnemonic activity. Such experiments are critical to clarify the impact of systemic NMDAR antagonists on WM representations, and to properly constrain computational models (Fig. 2). 
systemic application

(a)

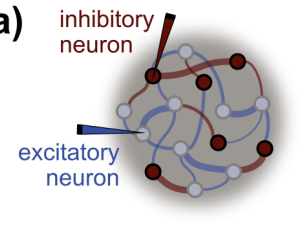

global NMDAR antagonist: preferentially affects

excitatory neurons
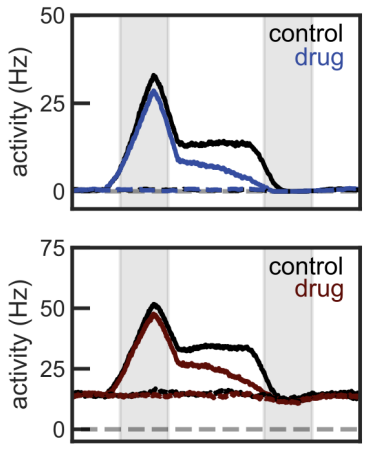

(b)

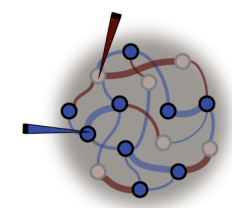

global NMDAR antagonist: preferentially affects inhibitory neurons
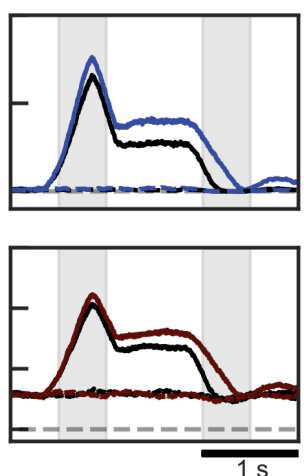

(c)

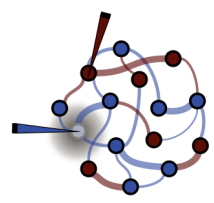

local NMDAR antagonist: applied to excitatory neuron
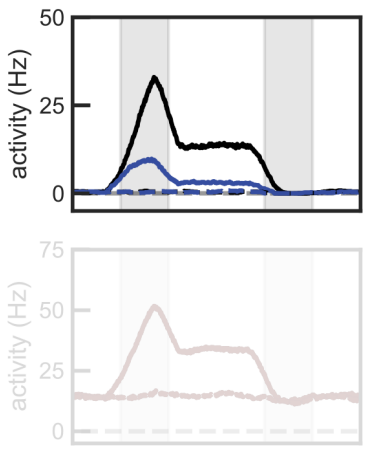

(d)

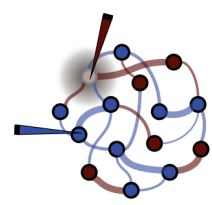

local NMDAR antagonist: applied to inhibitory neuron
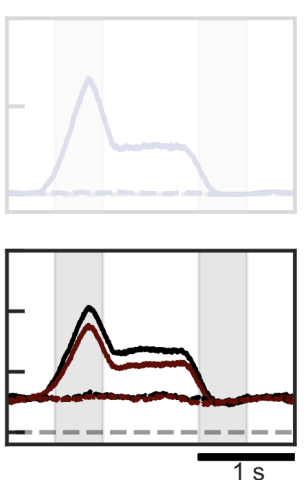

Figure 3. Dissociated effects of systemic vs. local application of NMDAR antagonists in a computational WM model. Depending on whether NMDAR blockade affects more strongly receptors on excitatory neurons than on inhibitory neurons, or vice versa, delay-period activity in network simulations gets reduced (a), or enhanced (b), for both excitatory (top graphs, blue) or inhibitory (bottom graphs, red) neurons. Instead, the local application of NMDAR antagonists invariably reduces delay-period activity in the affected neurons (c top, $d$ bottom) while global network dynamics remains unaltered, as reflected in the activity of non-modified neurons (c bottom, $d$ top). Details of network simulations are available at https://github.com/comptelab/attractorSz.

Based on currently available evidence, NMDAR antagonists would suppress prefrontal WM-related activity $[10,39,43,47,48]$. Recent evidence, however, also shows that WM deficits of PSZ may depend particularly on activity in parietal circuits [50]. Interestingly, NMDAR antagonists affect mnemonic activity in prefrontal and parietal circuits inversely [47], suggesting a possible enhancement of parietal WM-related activity. This contrast in the reaction of these two circuits to NMDAR manipulation is supported by marked differences in cellular physiological properties [51], which could impose different correlated noise structures (Fig. 2). Which of the two areas is more directly linked to WM behavioral output? There is evidence that prefrontal mnemonic activity reflects memory drifts that cause WM imprecisions [52,53], suggesting a closer link of this area to WM precision in SZ. However, it is not known if this relationship also exists for activity fluctuations in the parietal cortex, and which of the two areas is more closely associated with the activity drifts that drive WM imprecisions. As a result, specifying the dominant role of prefrontal or parietal circuits in maintaining accurate spatial WM signals through the mnemonic delay appears here as another key piece of information to constrain currently indecisive computational models (Fig. 2).

\section{Short-term plasticity deficits explain WM deficits in schizophrenia, without significant effects on E/l ratio}

On the basis of insufficiently constrained computational models, it is currently unclear whether regional imbalances in cortical E/l ratio caused by hypofunctional NMDARs can 
explain WM precision deficits in PSZ. However, computational models can unequivocally associate other possible mechanistic alterations with WM precision effects. Here, we consider a synaptic plasticity mechanism that explains reductions in WM precision in both attractor models considered in Figure 2. Schizophrenia has been related to dysfunctions in synaptic plasticity [54-57], and perceptual and neurocognitive alterations in the disease have been attributed to alterations in long- and short-term plasticity (STP) [58-60], also in relation to NMDAR hypofunction $[61,62]$. Cognitive effects of disrupted STP on WM have been assessed in genetic mouse models $[63,64]$ and computational models of schizophrenia [65], where reductions in STP lead to reduced WM performance [63,64]. Moreover, pharmacological inhibition of NMDARs through systemic ketamine administration decreases spike-time synchrony in delay-active cells in PFC during WM [66], which could be interpreted in light of reduced prefrontal STP [67]. Finally, computational modeling links a novel behavioral pattern of reduced serial dependence in PSZ and anti-NMDAR encephalitis to dysfunctional STP, while prefrontal E/l ratio alone could not account for these behavioral findings [29].

(a)

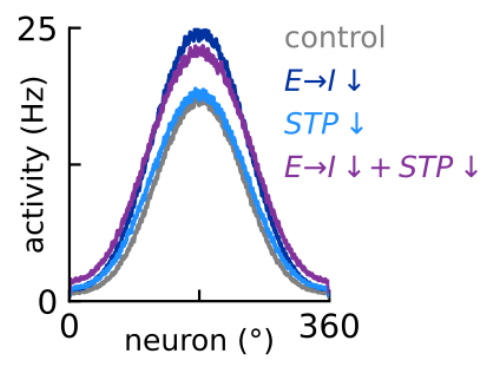

(b)

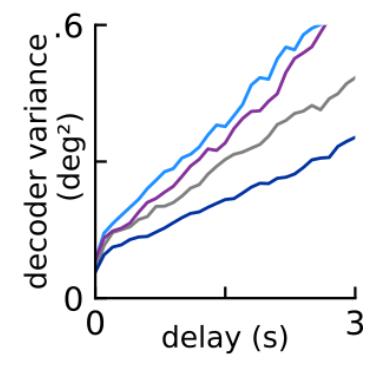

Figure 4. In network models, reductions of STP cause WM precision loss, also with concurrent E/I ratio alterations that would increase WM precision on their own. Simulations correspond to network Model 2 of Fig. 2. Control and E $\rightarrow \downarrow$ conditions are shared with Fig. 2, and are compared here with simulations with additional reduction in STP (STP $\downarrow$ and E $\rightarrow \downarrow+$ STP $\downarrow$, respectively). (a) Average delay-period activity of neurons in the network in each condition. Notice that STP reduction causes minimal changes in delay rates in the simulations, indicating quite intact $\mathrm{E} / \mathrm{l}$ ratio in the delay period after STP reduction. (b) Memory diffusion patterns for each condition (see Fig. 2d). Notice the strong impact of STP reduction on memory diffusion, to the point that it turns reduced diffusion of $E \rightarrow \mid \downarrow$ into enhanced diffusion ( $E \rightarrow \mid \downarrow+S T P \downarrow$ ) compared to control diffusion (gray).

The predictions for the effect of STP on WM are clear: when implemented in bump attractor networks, STP stabilizes bump attractors against noise, so that memory precision is better maintained through delay periods $[37,68,69]$, and bumps are more robust against intervening distractors [69]. Further, STP induces serial dependence between successive WM trials because of the enduring synaptic trace that influences subsequent bump attractor memories $[29,67,70,71]$. STP has these effects irrespectively of the specific network implementation of the bump attractor, and as a result its deficit leads unambiguously to WM precision loss (Fig. 4), increased distractibility [69] and reduced serial dependence [29], all characteristic WM alterations in PSZ (Box 1). Interestingly, this mechanism contributes weakly to delay-period activity, so its disruption could occur independently and in addition to mechanistic alterations that directly impact the E/I ratio (Fig. 4). For instance, assuming a network disinhibition in the circuit responsible for maintaining WM accuracy (possibly parietal cortex), Model 2 in Fig. 2 
would not be able to explain a reduction in WM precision through the alteration in E/I ratio but it could if in addition it had reduced STP (Fig. 4). Conversely, network inhibition as currently considered for prefrontal WM circuits in SZ would make Model 1 in Fig. 2 unable to account for WM precision loss, unless STP reductions are additionally considered (not shown). In fact, it is not unlikely that changes in neuromodulatory or neurotransmitter systems would affect both $\mathrm{E} / \mathrm{l}$ ratio and synaptic plasticity, with a potential interdependence of the two phenomena [72]. These scenarios may reconcile possible inconsistencies between experimental evidence and computational models of schizophrenia that are merely based on imbalances in E/l ratio (Fig. 2).

\section{Conclusions}

In this review, we argue that although contemporary network models of schizophrenia capture WM deficits observed in PSZ, the space of possible modeling solutions is not sufficiently constrained by experimental data. By focusing on the effect of increased vs. decreased $E / /$ ratio $(E \rightarrow \mid \downarrow$ vs. $E \rightarrow E \downarrow$ ) on WM precision, we provide two general insights. First, these opposed manipulations can produce the same behavioral pattern in different alternative, plausible biological network models (Fig. 2). As a result, the interpretations of mechanistic models in computational psychiatry will remain limited in the absence of properly constraining experimental data. Importantly, in-depth analysis of computational options can guide in the design of critically informative experiments. Second, we argue that grouping different perturbations under the convenient umbrella of E/I ratio can be inadequate in some conditions, as different pharmacological perturbations can affect the E/l ratio similarly, but have opposite effects on behavior [38,39].

In the specific case of how manipulations of the E/I ratio affect WM precision, we point out four important neurophysiological unknowns: The shape of activity bumps during WM delays, the specific properties of correlated noise in the circuit, the specific brain area responsible for maintaining accurate WM representations, and the possible joint alteration of NMDARs and STP. First, it appears critical to determine fine-grained effects of pharmacological interventions on neural tuning. For example, we showed that increases in the amplitude and width of tuning curves can affect WM precision in opposite directions, depending on the underlying computational model. Measuring tuning properties together with behavioral effects will be necessary to inform model selection. Second, spatial profiles of noise correlations may be critical to understand the effect of disease mechanisms on WM precision, and potentially other WM phenomena. Third, these neurophysiological characterizations should be done in the circuits most directly associated with maintaining precise WM, as current evidence comparing prefrontal and parietal cortices shows that they respond very differently to NMDAR antagonists. Pharmacological experiments in vivo will be key to inform computational models of schizophrenia by systematically measuring these fine-grained neurophysiological effects directly in the area responsible for WM maintenance. Finally, in vitro experiments should determine if NMDAR antagonists also impact slower mechanisms (e.g. different types of STP), as computational models unequivocally assign a memory-stabilizing role to such slower mechanisms and this could interact with interpretations of WM precision deficits in PSZ (Fig. 4).

There is also pending work on the computational and theoretical front. Shared correlations, in addition to the commonly considered bump attractor shape [19], impact WM precision 
[32], and only recently have studies started to address how these shared correlations are generated in recurrently coupled networks $[35,36]$. These efforts should continue and extend also to structured attractor states, eventually aiming to understand the role of biophysical mechanisms that have been implicated in schizophrenia such as NMDARs, dopamine or STP. Furthermore, PSZ show abnormal behavioral patterns in other cognitive tasks that require context-dependent, cognitive control and have been linked to altered $\mathrm{E} / \mathrm{I}$ ratio [73-75]. Developing biophysical computational models would allow the mechanistic understanding of these deficits through the consistent integration of computational and neurophysiology data, as attempted here for spatial WM precision. Recurrent neural networks (RNNs) trained with backpropagation are a possible tool to address more complex tasks that are difficult to model bottom-up [76]. Training RNNs to replicate behavioral patterns and neural dynamics in PSZ, or pharmacological models of schizophrenia, could untie different contributions of El-structured connectivity (Ehrlich et al. abstract 1-052, Computational and Systems Neuroscience (Cosyne) Conference, February 2021) [77,78] and STP [79], which appear as the key ingredients to understand WM deficits, and perhaps cognition more broadly, in PSZ.

Finally, we want to acknowledge the contribution of computational models of disease to the understanding of neuropsychiatric disorders. While pointing out inadequacies of current models to provide a consistent account of cognitive deficits in PSZ, we also showed how the analysis of these models allows a more precise framing of the research questions to advance concertedly towards a mechanistic understanding of these diseases. This underscores the rigour that mathematical modeling adds to the field of psychiatry, and we hope that it encourages clinical researchers to adopt these models as a formal framework to understand disease. 


\section{Box 1. Quantitative WM deficits associated with schizophrenia}

Precision. PSZ exhibit loss of WM precision [28,80-82], especially for long memory delays [81]. In computational models, this effect can be accounted for by increased E/I ratio due to NMDAR hypofunction at interneurons [19,28,29,81], decreased E/I ratio due to NMDAR hypofunction at pyramidal neurons (Fig. 1), dopaminergic imbalances [83], or the disruption of STP $[29,69]$.

Distractibility. Memory reports of PSZ, relative to healthy controls, are substantially more biased towards distractors $[28,81]$. This effect has been simulated in network models with increased E/I ratio [28], decreased D1/D2 ratio [20,84,85], and disrupted STP [69]. In addition to enhanced biases towards distractors, PSZ also exhibit occasional substantial repulsion from nearby distractors [28,81]. This effect cannot be accounted for by attractor models for WM [81], but it is still unclear if it is a delay-dependent effect or it reflects alterations in perceptual processing. Indeed, experimental protocols that separate perceptual and WM origins (by including a 0-delay condition, for instance) when measuring distractibility [86] and serial dependence $[29,87-89]$ in healthy controls have suggested a perceptual origin of similar repulsive biases.

Capacity. Reduced WM capacity characterizes PSZ [90-93]. Computational models show that WM capacity may be determined by multiple factors: interference between memories [86,94-96], memory forgetting [95-97], feature-binding errors or swap errors [98], and the spontaneous generation of false memories [21]. Some of these factors have been reported to be affected in PSZ, like increased incidence of false memories [99], but more studies are needed to better specify the origin of reduced WM capacity in PSZ. In line with all these different factors, also multiple biological mechanisms may underlie WM capacity deficits in SZ: reduced E/I ratio [33,95,97], dopaminergic imbalances [83], reduced efficacy of top-down inputs [97], functional cortico-cortical disconnection [96], impaired oscillatory dynamics $[100,101]$. More sophisticated experiments and appropriate data analyses will be key to disentangle the different origins and mechanisms of reduced WM capacity in PSZ.

Serial dependence or proactive interference. Tasks engaging WM induce across trial interference, in what is commonly called serial dependence [102]. In contrast with the biases previously described in this box, serial dependence is reduced in PSZ [29] intriguingly, this reduction also characterizes autism [103]. Attractor models with STP have been proposed for serial dependence [70,71], and it has been shown that, in these models, alterations in STP, but not in the E/I ratio of the attractor models, can account for reduced serial dependence in PSZ [29]. 


\section{Acknowledgments}

We acknowledge support from Institute Carlos III, Spain (Ref: PIE 16/00014), CELLEX Foundation, Safra Foundation, CERCA Programme/Generalitat de Catalunya, Generalitat de Catalunya (AGAUR 2014SGR1265, 2017SGR01565), "la Caixa" Foundation (ID 100010434, under the agreement LCF/PR/HR17/52150001), and by the Spanish Ministry of Science, Competitiveness and Universities co-funded by the European Regional Development Fund (Refs: BFU 2015-65318-R, RTI2018-094190-B-I00). We thank the Barcelona Supercomputing Center (BSC) for providing computing resources. We thank Jaime de la Rocha, Zachary Kilpatrick, Alexander Seeholzer, Alexandre Mahrach and Peter Murphy for feedback and discussions.

\section{Highlighted references}

** Darshan R, et al. (2018) Phys. Rev. X, 8:031072

This elegant computational study explores the network mechanisms that can support strong shared variability in large networks of irregularly firing neurons. This pioneering work starts exploring a largely unknown aspect of recurrent networks, especially in relation to attractor states as required for working memory.

* Gonzalez-Burgos et al. (2019) J. Neurosci, 29:7277-7290

Gonzales-Burgos et al. compare for the first time physiological properties and the dendrite morphology of layer 3 pyramidal neurons in prefrontal and posterior parietal cortex. Prefrontal neurons are physiologically more diverse, with a large proportion of bursty neurons. This suggests fundamental differences in how these two areas engage in reverberant network dynamics during working memory maintenance; in particular, their mechanisms of shared correlations, which may play an important role in understanding WM precision deficits in PSZ.

** Gold, J. M., et al. (2020). Biol. Psychiatry Cogn. Neurosci. Neuroimaging 5, 913-922.

Using a large cohort and an extensive parametric task design, Gold et al. consolidate previous findings of spatial working memory deficits in schizophrenia, namely higher distractibility and memory diffusion, and show their consistency with a computational network model. Similar parametric studies with NMDAR-antagonists to test the glutamatergic basis of these deficits in PSZ (Box 1) are still missing..

** Kummerfeld et al. (2020) Biol. Psychiatry Cogn. Neurosci. Neuroimaging, 5:705-714.

As part of a series of recent papers reporting on elegant and difficult experiments with monkeys performing a complex working memory task (DPX task) under systemic ketamine administration, with simultaneous prefrontal and parietal recordings, Kummerfeld et al. show that NMDAR blockade influences prefrontal and parietal networks in opposing ways during WM. This intriguing result questions hypotheses currently implemented in computational models, which typically assume the general disinhibition of cortical circuits.

* Lieder, Adam et al. (2019) Nat. Neurosci. 2019, 22:256-264.

Lieder, Adam et al. found that serial dependence is reduced in people with autism, but not in dyslexic individuals, while this last group shows a reduced impact of long-term stimulus statistics. This is the first study showing reduced serial dependence in clinical populations. 
That serial dependence is also reduced in schizophrenia suggests a potential link between the two diseases.

* Masse, N. Y., et al. (2019). Nat. Neurosci. 22, 1159-1167.

Masse et al. trained recurrent neural networks to find how working memory can be supported by persistent firing or short-term plasticity, dependending on the task characteristics. This approach is a promising avenue for computational psychiatry in general, and the study of working memory deficits in schizophrenia in particular.

** Qi, X.-L., et al. (2019). BioRxiv.

Qi et al. provide experimental validation of the impact of bump attractor shape on working memory diffusivity (Eq. 1). They manipulate the cholinergic neuromodulation system by microstimulation of nucleus basalis and show that working memory precision increases, and bump attractors in the prefrontal cortex become wider. This finding is consistent with an attractor network model. The contrast of these results with those of Roussy et al. (2020) prompt some of the discussions in this review.

** Roussy et al. (2020) BioRxiv, doi:10.1101/2020.06.19.162446.

In a unique study of systemic administration of ketamine in monkeys performing complex spatial working memory tasks, Roussy et al offer evidence for disinhibition as well as decreased working memory precision. The contrast of these results with those of Qi et al. (2019) prompt some of the discussions in this review.

* Seeholzer, A. et al. (2019) PLoS Comput. Biol. 2019, 15:e1006928.

Although not explicitly related to schizophrenia, Seeholzer et al present a comprehensive and mechanistic explanation of how short-term plasticity improves working memory function in attractor network models, which can directly inform deficits caused by a disruption of short-term plasticity.

** Stein, H., Barbosa, J., et al. (2020). Nat. Commun. 11, 4250.

Stein, Barbosa et al. found that delay-dependent serial dependence in working memory is strikingly reduced in people with schizophrenia and anti-NMDAR encephalitis, compared to healthy controls. Through biophysical modeling, the authors propose a reduction of short-term plasticity to account for the finding.

* van Vugt, B., et al. (2020). J. Neurosci. 40, 2458-2470.

Using iontophoresis to compare the effects of NMDAR- and AMPAR-antagonists on delay firing, van Vugt et al. show that an inhibition of NMDAR leads to a reduction of tuned persistent activity, while the inhibition of AMPAR lead to a more general reduction in firing rates. Together with earlier work by Amy Arnsten, this confirms the strong role of NMDARs in maintaining tuned persistent activity in working memory, and its vulnerability in mental diseases affecting NMDARs. 


\section{Bibliography}

1. Wang X-J, Krystal JH: Computational psychiatry. Neuron 2014, 84:638-654.

2. Huys QJM, Browning M, Paulus MP, Frank MJ: Advances in the computational understanding of mental illness. Neuropsychopharmacology 2020, 46:3-19.

3. Seriès P: Computational Psychiatry: A Primer. The MIT Press; 2020.

4. Deco G, Kringelbach ML: Great expectations: using whole-brain computational connectomics for understanding neuropsychiatric disorders. Neuron 2014, 84:892-905.

5. Anticevic A, Murray JD, Barch DM: Bridging levels of understanding in schizophrenia through computational modeling. Clin. Psychol. Sci. 2015, 3:433-459.

6. Bennett $D$, Silverstein SM, Niv Y: The two cultures of computational psychiatry. JAMA Psychiatry 2019, 76:563-564.

7. Valton V, Romaniuk L, Douglas Steele J, Lawrie S, Seriès P: Comprehensive review: Computational modelling of schizophrenia. Neurosci. Biobehav. Rev. 2017, 83:631-646.

8. Krystal JH, Anticevic A, Yang GJ, Dragoi G, Driesen NR, Wang X-J, Murray JD: Impaired tuning of neural ensembles and the pathophysiology of schizophrenia: A translational and computational neuroscience perspective. Biol. Psychiatry 2017, 81:874-885.

9. Hamm JP, Peterka DS, Gogos JA, Yuste R: Altered cortical ensembles in mouse models of schizophrenia. Neuron 2017, 94:153-167.e8.

10. Wang M, Yang Y, Wang C-J, Gamo NJ, Jin LE, Mazer JA, Morrison JH, Wang X-J, Arnsten AFT: NMDA receptors subserve persistent neuronal firing during working memory in dorsolateral prefrontal cortex. Neuron 2013, 77:736-749.

11. Jackson ME, Homayoun $\mathrm{H}$, Moghaddam $B$ : NMDA receptor hypofunction produces concomitant firing rate potentiation and burst activity reduction in the prefrontal cortex. Proc Natl Acad Sci USA 2004, 101:8467-8472.

12. Pafundo DE, Miyamae T, Lewis DA, Gonzalez-Burgos G: Presynaptic Effects of N-Methyl-D-Aspartate Receptors Enhance Parvalbumin Cell-Mediated Inhibition of Pyramidal Cells in Mouse Prefrontal Cortex. Biol. Psychiatry 2018, 84:460-470.

13. Wang Y, Markram H, Goodman PH, Berger TK, Ma J, Goldman-Rakic PS: Heterogeneity in the pyramidal network of the medial prefrontal cortex. Nat. Neurosci. 2006, 9:534-542.

14. Durstewitz D, Huys QJM, Koppe G: Psychiatric illnesses as disorders of network dynamics. Biol. Psychiatry Cogn. Neurosci. Neuroimaging 2020, doi:10.1016/j.bpsc.2020.01.001.

15. Green IW, Glausier JR: Different paths to core pathology: the equifinal model of 
the schizophrenia syndrome. Schizophr. Bull. 2016, 42:542-549.

16. Loh M, Rolls ET, Deco G: A dynamical systems hypothesis of schizophrenia. PLoS Comput. Biol. 2007, 3:e228.

17. Rolls ET, Loh M, Deco G, Winterer G: Computational models of schizophrenia and dopamine modulation in the prefrontal cortex. Nat. Rev. Neurosci. 2008, 9:696-709.

18. Tanaka S: Dopaminergic control of working memory and its relevance to schizophrenia: a circuit dynamics perspective. Neuroscience 2006, 139:153-171.

19. Murray JD, Anticevic A, Gancsos M, Ichinose M, Corlett PR, Krystal JH, Wang X-J: Linking microcircuit dysfunction to cognitive impairment: effects of disinhibition associated with schizophrenia in a cortical working memory model. Cereb. Cortex 2014, 24:859-872.

20. Durstewitz D, Seamans JK, Sejnowski TJ: Dopamine-mediated stabilization of delay-period activity in a network model of prefrontal cortex. J. Neurophysiol. 2000, 83:1733-1750.

21. Cano-Colino M, Compte A: A computational model for spatial working memory deficits in schizophrenia. Pharmacopsychiatry 2012, 45 Suppl 1:S49-56.

22. Lisman J: Excitation, inhibition, local oscillations, or large-scale loops: what causes the symptoms of schizophrenia? Curr. Opin. Neurobiol. 2012, 22:537-544.

23. Grent-'t-Jong T, Gross J, Goense J, Wibral M, Gajwani R, Gumley Al, Lawrie SM, Schwannauer M, Schultze-Lutter F, Navarro Schröder T, et al.: Resting-state gamma-band power alterations in schizophrenia reveal E/l-balance abnormalities across illness-stages. elife 2018, 7.

24. Anticevic A, Gancsos M, Murray JD, Repovs G, Driesen NR, Ennis DJ, Niciu MJ, Morgan PT, Surti TS, Bloch MH, et al.: NMDA receptor function in large-scale anticorrelated neural systems with implications for cognition and schizophrenia. Proc Natl Acad Sci USA 2012, 109:16720-16725.

25. Nakazawa K, Sapkota K: The origin of NMDA receptor hypofunction in schizophrenia. Pharmacol. Ther. 2020, 205:107426.

26. Lisman JE, Coyle JT, Green RW, Javitt DC, Benes FM, Heckers S, Grace AA: Circuit-based framework for understanding neurotransmitter and risk gene interactions in schizophrenia. Trends Neurosci. 2008, 31:234-242.

27. Bygrave AM, Kilonzo K, Kullmann DM, Bannerman DM, Kätzel D: Can N-Methyl-D-Aspartate Receptor Hypofunction in Schizophrenia Be Localized to an Individual Cell Type? Front. Psychiatry 2019, 10:835.

28. Starc M, Murray JD, Santamauro N, Savic A, Diehl C, Cho YT, Srihari V, Morgan PT, Krystal $\mathrm{JH}$, Wang $\mathrm{X}-\mathrm{J}$, et al.: Schizophrenia is associated with a pattern of spatial working memory deficits consistent with cortical disinhibition. Schizophr. Res. 2017, 181:107-116.

29. Stein H, Barbosa J, Rosa-Justicia M, Prades L, Morató A, Galan-Gadea A, Ariño H, 
Martinez-Hernandez E, Castro-Fornieles J, Dalmau J, et al.: Reduced serial dependence suggests deficits in synaptic potentiation in anti-NMDAR encephalitis and schizophrenia. Nat. Commun. 2020, 11:4250.

30. Cavanagh SE, Lam NH, Murray JD, Hunt LT, Kennerley SW: A circuit mechanism for decision-making biases and NMDA receptor hypofunction. elife 2020, 9.

31. Amari S: Dynamics of pattern formation in lateral-inhibition type neural fields. Biol. Cybern. 1977, 27:77-87.

32. Kilpatrick ZP, Ermentrout $\mathrm{B}$ : Wandering bumps in stochastic neural fields. SIAM J. Appl. Dyn. Syst. 2013, 12:61-94.

33. Krishnan N, Poll DB, Kilpatrick ZP: Synaptic efficacy shapes resource limitations in working memory. J. Comput. Neurosci. 2018, 44:273-295.

34. Shaham N, Burak Y: Slow diffusive dynamics in a chaotic balanced neural network. PLoS Comput. Biol. 2017, 13:e1005505.

35. Rosenbaum R, Smith MA, Kohn A, Rubin JE, Doiron B: The spatial structure of correlated neuronal variability. Nat. Neurosci. 2017, 20:107-114.

36. Darshan R, van Vreeswijk C, Hansel D: Strength of correlations in strongly recurrent neuronal networks. Phys. Rev. X 2018, 8:031072.

37. Hansel D, Mato G: Short-term plasticity explains irregular persistent activity in working memory tasks. J. Neurosci. 2013, 33:133-149.

38. Qi X-L, Liu R, Vazdarjanova Al, Blake DT, Constantinidis C: Nucleus basalis stimulation stabilizes attractor networks and enhances task representation in prefrontal cortex. BioRxiv 2019, doi:10.1101/674465.

39. Roussy M, Luna R, Duong L, Corrigan B, Gulli RA, Nogueira R, Moreno-Bote R, Sachs AJ, Palaniyappan L, Martinez-Trujillo JC: Naturalistic coding of working memory in primate prefrontal cortex. BioRxiv 2020, doi:10.1101/2020.06.19.162446.

40. Ma L, Skoblenick K, Seamans JK, Everling S: Ketamine-Induced Changes in the Signal and Noise of Rule Representation in Working Memory by Lateral Prefrontal Neurons. J. Neurosci. 2015, 35:11612-11622.

41. Ichinose $M$, Park S: Mechanisms underlying visuospatial working memory impairments in schizophrenia. Curr. Top. Behav. Neurosci. 2019, 41:345-367.

42. Beck K, Hindley G, Borgan F, Ginestet C, McCutcheon R, Brugger S, Driesen N, Ranganathan M, D'Souza DC, Taylor M, et al.: Association of Ketamine With Psychiatric Symptoms and Implications for Its Therapeutic Use and for Understanding Schizophrenia: A Systematic Review and Meta-analysis. JAMA Netw. Open 2020, 3:e204693.

43. Driesen NR, McCarthy G, Bhagwagar Z, Bloch MH, Calhoun VD, D'Souza DC, Gueorguieva R, He G, Leung H-C, Ramani R, et al.: The impact of NMDA receptor blockade on human working memory-related prefrontal function and connectivity. Neuropsychopharmacology 2013, 38:2613-2622.

44. Moghaddam B, Adams B, Verma A, Daly D: Activation of glutamatergic neurotransmission by ketamine: a novel step in the pathway from NMDA 
receptor blockade to dopaminergic and cognitive disruptions associated with the prefrontal cortex. J. Neurosci. 1997, 17:2921-2927.

45. Driesen NR, Leung H-C, Calhoun VD, Constable RT, Gueorguieva R, Hoffman R, Skudlarski P, Goldman-Rakic PS, Krystal JH: Impairment of working memory maintenance and response in schizophrenia: functional magnetic resonance imaging evidence. Biol. Psychiatry 2008, 64:1026-1034.

46. Glahn DC, Ragland JD, Abramoff A, Barrett J, Laird AR, Bearden CE, Velligan DI: Beyond hypofrontality: a quantitative meta-analysis of functional neuroimaging studies of working memory in schizophrenia. Hum. Brain Mapp. 2005, 25:60-69.

47. Kummerfeld E, Ma S, Blackman RK, DeNicola AL, Redish AD, Vinogradov S, Crowe $\mathrm{DA}$, Chafee MV: Cognitive control errors in nonhuman primates resembling those in schizophrenia reflect opposing effects of NMDA receptor blockade on causal interactions between cells and circuits in prefrontal and parietal cortices. Biol. Psychiatry Cogn. Neurosci. Neuroimaging 2020, 5:705-714.

48. Yang S, Seo H, Wang M, Arnsten AFT: NMDAR neurotransmission needed for persistent neuronal firing: Potential roles in mental disorders. Frontiers in Psychiatry 2021, [no volume].

49. van Vugt B, van Kerkoerle T, Vartak D, Roelfsema PR: The contribution of AMPA and NMDA receptors to persistent firing in the dorsolateral prefrontal cortex in working memory. J. Neurosci. 2020, 40:2458-2470.

50. Hahn B, Robinson BM, Leonard CJ, Luck SJ, Gold JM: Posterior parietal cortex dysfunction is central to working memory storage and broad cognitive deficits in schizophrenia. J. Neurosci. 2018, 38:8378-8387.

51. González-Burgos G, Miyamae T, Krimer Y, Gulchina Y, Pafundo DE, Krimer O, Bazmi $\mathrm{H}$, Arion D, Enwright JF, Fish KN, et al.: Distinct Properties of Layer 3 Pyramidal Neurons from Prefrontal and Parietal Areas of the Monkey Neocortex. J. Neurosci. 2019, 39:7277-7290.

52. Wimmer K, Nykamp DQ, Constantinidis C, Compte A: Bump attractor dynamics in prefrontal cortex explains behavioral precision in spatial working memory. Nat. Neurosci. 2014, 17:431-439.

53. Sajad A, Sadeh M, Yan X, Wang H, Crawford JD: Transition from Target to Gaze Coding in Primate Frontal Eye Field during Memory Delay and Memory-Motor Transformation. Eneuro 2016, 3.

54. Hall J, Trent S, Thomas KL, O'Donovan MC, Owen MJ: Genetic risk for schizophrenia: convergence on synaptic pathways involved in plasticity. Biol. Psychiatry 2015, 77:52-58.

55. De Picker LJ, Morrens M, Chance SA, Boche D: Microglia and Brain Plasticity in Acute Psychosis and Schizophrenia Illness Course: A Meta-Review. Front. Psychiatry 2017, 8:238.

56. Nanou E, Catterall WA: Calcium channels, synaptic plasticity, and neuropsychiatric disease. Neuron 2018, 98:466-481.

57. Averbeck BB, Chafee MV: Using model systems to understand errant plasticity 
mechanisms in psychiatric disorders. Nat. Neurosci. 2016, 19:1418-1425.

58. Thakkar KN, Antinori A, Carter OL, Brascamp JW: Altered short-term neural plasticity related to schizotypal traits: Evidence from visual adaptation. Schizophr. Res. 2019, 207:48-57.

59. Jahshan C, Wynn JK, Mathalon DH, Green MF: Cognitive correlates of visual neural plasticity in schizophrenia. Schizophr. Res. 2017, 190:39-45.

60. Forsyth JK, Lewis DA: Mapping the Consequences of Impaired Synaptic Plasticity in Schizophrenia through Development: An Integrative Model for Diverse Clinical Features. Trends Cogn Sci (Regul Ed) 2017, 21:760-778.

61. Kantrowitz JT: $\mathbf{N}$-methyl-d-aspartate-type glutamate receptor modulators and related medications for the enhancement of auditory system plasticity in schizophrenia. Schizophr. Res. 2019, 207:70-79.

62. Kantrowitz JT, Swerdlow NR, Dunn W, Vinogradov S: Auditory System Target Engagement During Plasticity-Based Interventions in Schizophrenia: A Focus on Modulation of N-Methyl-D-Aspartate-Type Glutamate Receptor Function. Biol. Psychiatry Cogn. Neurosci. Neuroimaging 2018, 3:581-590.

63. Arguello PA, Gogos JA: Genetic and cognitive windows into circuit mechanisms of psychiatric disease. Trends Neurosci. 2012, 35:3-13.

64. Crabtree GW, Gogos JA: Synaptic plasticity, neural circuits, and the emerging role of altered short-term information processing in schizophrenia. Front. Synaptic Neurosci. 2014, 6:28.

65. Becker S, Nold A, Tchumatchenko T: Formation and synaptic control of active transient working memory representations. BioRxiv 2020, doi:10.1101/2020.08.30.273995.

66. Zick JL, Blackman RK, Crowe DA, Amirikian B, DeNicola AL, Netoff TI, Chafee MV: Blocking NMDAR Disrupts Spike Timing and Decouples Monkey Prefrontal Circuits: Implications for Activity-Dependent Disconnection in Schizophrenia. Neuron 2018, 98:1243-1255.e5.

67. Barbosa J, Stein H, Martinez RL, Galan-Gadea A, Li S, Dalmau J, Adam KCS, Valls-Solé J, Constantinidis C, Compte A: Interplay between persistent activity and activity-silent dynamics in the prefrontal cortex underlies serial biases in working memory. Nat. Neurosci. 2020, 23:1016-1024.

68. Itskov V, Hansel D, Tsodyks M: Short-Term Facilitation may Stabilize Parametric Working Memory Trace. Front. Comput. Neurosci. 2011, 5:40.

69. Seeholzer A, Deger M, Gerstner W: Stability of working memory in continuous attractor networks under the control of short-term plasticity. PLoS Comput. Biol. 2019, 15:e1006928.

70. Kilpatrick ZP: Synaptic mechanisms of interference in working memory. Sci. Rep. 2018, 8:7879.

71. Bliss DP, D'Esposito M: Synaptic augmentation in a cortical circuit model reproduces serial dependence in visual working memory. PLOS ONE 2017, 12:e0188927. 
72. Meunier CNJ, Chameau P, Fossier PM: Modulation of synaptic plasticity in the cortex needs to understand all the players. Front. Synaptic Neurosci. 2017, 9:2.

73. Jones JAH, Sponheim SR, MacDonald AW: The dot pattern expectancy task: reliability and replication of deficits in schizophrenia. Psychol. Assess. 2010, 22:131-141.

74. Jardri R, Duverne S, Litvinova AS, Denève S: Experimental evidence for circular inference in schizophrenia. Nat. Commun. 2017, 8:14218.

75. Blackman RK, Macdonald AW, Chafee MV: Effects of ketamine on context-processing performance in monkeys: a new animal model of cognitive deficits in schizophrenia. Neuropsychopharmacology 2013, 38:2090-2100.

76. Yang GR, Wang X-J: Artificial neural networks for neuroscientists: A primer. Neuron 2020, 107:1048-1070.

77. Song HF, Yang GR, Wang X-J: Training Excitatory-Inhibitory Recurrent Neural Networks for Cognitive Tasks: A Simple and Flexible Framework. PLoS Comput. Biol. 2016, 12:e1004792.

78. Kim R, Sejnowski TJ: Strong inhibitory signaling underlies stable temporal dynamics and working memory in spiking neural networks. Nat. Neurosci. 2021, 24:129-139.

79. Masse NY, Yang GR, Song HF, Wang X-J, Freedman DJ: Circuit mechanisms for the maintenance and manipulation of information in working memory. Nat. Neurosci. 2019, 22:1159-1167.

80. Xie W, Cappiello M, Park H-B, Deldin P, Chan RCK, Zhang W: Schizotypy is associated with reduced mnemonic precision in visual working memory. Schizophr. Res. 2018, 193:91-97.

81. Gold JM, Bansal S, Anticevic A, Cho YT, Repovš G, Murray JD, Hahn B, Robinson $B M$, Luck SJ: Refining the empirical constraints on computational models of spatial working memory in schizophrenia. Biol. Psychiatry Cogn. Neurosci. Neuroimaging 2020, 5:913-922.

82. Badcock JC, Badcock DR, Read C, Jablensky A: Examining encoding imprecision in spatial working memory in schizophrenia. Schizophr. Res. 2008, 100:144-152.

83. Okimura T, Tanaka S, Maeda T, Kato M, Mimura M: Simulation of the capacity and precision of working memory in the hypodopaminergic state: Relevance to schizophrenia. Neuroscience 2015, 295:80-89.

84. Durstewitz D, Seamans JK: The dual-state theory of prefrontal cortex dopamine function with relevance to catechol-o-methyltransferase genotypes and schizophrenia. Biol. Psychiatry 2008, 64:739-749.

85. Brunel $\mathrm{N}$, Wang $\mathrm{XJ}$ : Effects of neuromodulation in a cortical network model of object working memory dominated by recurrent inhibition. J. Comput. Neurosci. 2001, 11:63-85.

86. Almeida R, Barbosa J, Compte A: Neural circuit basis of visuo-spatial working memory precision: a computational and behavioral study. J. Neurophysiol. 2015, 114:1806-1818. 
87. Bliss DP, Sun JJ, D'Esposito M: Serial dependence is absent at the time of perception but increases in visual working memory. Sci. Rep. 2017, 7:14739.

88. Fritsche $M$, Mostert $P$, de Lange FP: Opposite effects of recent history on perception and decision. Curr. Biol. 2017, 27:590-595.

89. Pascucci D, Mancuso G, Santandrea E, Della Libera C, Plomp G, Chelazzi L: Laws of concatenated perception: Vision goes for novelty, decisions for perseverance. PLoS Biol. 2019, 17:e3000144.

90. Johnson MK, McMahon RP, Robinson BM, Harvey AN, Hahn B, Leonard CJ, Luck SJ, Gold JM: The relationship between working memory capacity and broad measures of cognitive ability in healthy adults and people with schizophrenia. Neuropsychology 2013, 27:220-229.

91. Gold JM, Hahn B, Zhang WW, Robinson BM, Kappenman ES, Beck VM, Luck SJ: Reduced capacity but spared precision and maintenance of working memory representations in schizophrenia. Arch. Gen. Psychiatry 2010, 67:570-577.

92. Mayer JS, Fukuda K, Vogel EK, Park S: Impaired contingent attentional capture predicts reduced working memory capacity in schizophrenia. PLOS ONE 2012, 7:e48586.

93. Collins AGE, Brown JK, Gold JM, Waltz JA, Frank MJ: Working memory contributions to reinforcement learning impairments in schizophrenia. $J$. Neurosci. 2014, 34:13747-13756.

94. Nassar MR, Helmers JC, Frank MJ: Chunking as a rational strategy for lossy data compression in visual working memory. Psychol. Rev. 2018, 125:486-511.

95. Wei Z, Wang X-J, Wang D-H: From distributed resources to limited slots in multiple-item working memory: a spiking network model with normalization. $J$. Neurosci. 2012, 32:11228-11240.

96. Bouchacourt F, Buschman TJ: A flexible model of working memory. Neuron 2019, 103:147-160.e8.

97. Edin F, Klingberg T, Johansson P, McNab F, Tegnér J, Compte A: Mechanism for top-down control of working memory capacity. Proc Natl Acad Sci USA 2009, 106:6802-6807.

98. Schneegans S, Bays PM: No fixed item limit in visuospatial working memory. Cortex 2016, 83:181-193.

99. Mayer JS, Park S: Working memory encoding and false memory in schizophrenia and bipolar disorder in a spatial delayed response task. $J$. Abnorm. Psychol. 2012, 121:784-794.

100. Pina JE, Bodner M, Ermentrout B: Oscillations in working memory and neural binding: A mechanism for multiple memories and their interactions. PLOS Comput. Biol. 2018, 14:e1006517.

101. Barbosa J, Sreenivasan $\mathrm{K}$, Compte A: Feature-binding in working memory through neuronal synchronization. In 2019 Conference on Cognitive Computational Neuroscience. Edited by Cognitive Computational Neuroscience. Cognitive Computational Neuroscience; 2019. 
102. Kiyonaga A, Scimeca JM, Bliss DP, Whitney D: Serial Dependence across

Perception, Attention, and Memory. Trends Cogn Sci (Regul Ed) 2017, 21:493-497.

103. Lieder I, Adam V, Frenkel O, Jaffe-Dax S, Sahani M, Ahissar M: Perceptual bias reveals slow-updating in autism and fast-forgetting in dyslexia. Nat. Neurosci. 2019, 22:256-264. 